\title{
Ordenar as Universidades IBero-Americanas: QUANTIDADE, QUALIDADE E DIMENSÃO
}

\author{
Susana Jarmelo1, Tânia F. G. G. Cova², Alberto A. C. C. Pais²,3, J. Sérgio Seixas de Melo ${ }^{1,2,3^{*}}$, \\ Sebastião J. Formosinho2,3 \\ ${ }^{1}$ Faculdade de Ciências e Tecnologia da Universidade de Coimbra \\ ${ }^{2}$ Departamento de Química, Faculdade de Ciências e Tecnologia da Universidade de Coimbra \\ ${ }^{3}$ Centro de Química de Coimbra, Universidade de Coimbra \\ sseixas@ci.uc.pt
}

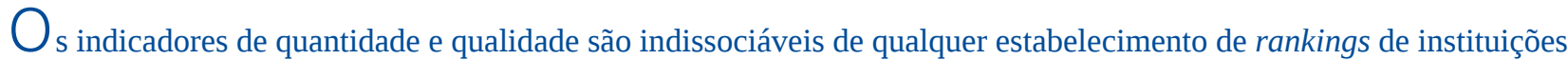
geradoras de conhecimento científico. Neste estudo é feita uma análise comparativa da produção científica de 51 instituições de Ensino Superior no universo ibero-americano tendo em conta dados provenientes do ranking SCImago, aferidos das bases Thomson ISI Web of Science (WoS) e Essential Science Indicators (ESI). Especificamente, o estudo contempla o tratamento e interpretação crítica dos dados da ciência produzida em seis países ibero-americanos (Portugal, Espanha, Brasil, Argentina, México e Chile) e estabelece critérios que perspectivam a classificação das suas instituições.
\end{abstract}

\section{INTRODUÇÃO}

A análise da produção científica e o reconhecimento dos investigadores junto da comunidade científica assentam, em grande medida, na aplicação de diversos indicadores bibliométricos, que quantificam o seu impacto científico. Estes indicadores acompanham o desenvolvimento das diversas áreas científicas e são realimentados pelos próprios resultados de investigação. Numa perspectiva individual, a fórmula de Hirsch, publicada em 2005 [1], avalia os investigadores, combinando a produtividade traduzida pelo número de artigos e o impacto da pesquisa, através do número de citações. Possivelmente, uma das razões pelas quais este índice se afirmou tão rapidamente, a despeito de críticas e sugestões alternativas promovidas pelo próprio sucesso, tem a ver com o facto de combinar quantidade e qualidade. Esta combinação é bem mais difícil de conseguir nas avaliações de carácter institucional. Mas é possível, num dado período, estimar o índice-h de uma instituição numa dada área científica.

Existem, actualmente, várias bases de dados que disponibilizam indicadores e resultados de análise bibliométrica, sendo a mais conhecida a Web of Science (WoS) da Thomson Reuteurs. No entanto, existem outras fontes e ferramentas que também fornecem dados com base nas citações, como a Scopus da Elsevier e a Google Scholar Metrics (GSM).

A validade da aplicação dos indicadores de produtividade científica tem sido alvo de crítica por parte de vários autores. Em Portugal, esta discussão ganhou visibilidade em 2005 quando o jornal Público disponibilizou um extenso trabalho sobre rankings das universidades portuguesas baseado num estudo de Luís Sousa Lobo (antigo Reitor da Universidade Nova de Lisboa), entregue ao Conselho de Reitores das Universidades Portuguesas (CRUP) e que circularia nos meios académicos portugueses [2]. $\mathrm{O}$ estudo, baseado num ratio entre a produção científica bruta total (retirada da base de dados Thomson ISI Web of Science que na altura se tornara disponível à comunidade académica portuguesa) e o número de docentes de carreira das diferentes universidades portuguesas, colocava as Universidades de Aveiro e do Algarve, cujo ratio seria mais elevado, mais próximas das universidades consideradas "Universidades de Investigação". Em termos globais de produção (número de artigos) seriam as Universidades do Porto e Técnica de Lisboa que lideravam. O estudo, caracterizado por alguma controvérsia, conduziu a inúmeros comentários de cientistas e reitores, alguns publicados nos dias seguintes no mesmo jornal.

A questão da avaliação da qualidade da ciência produzida pode parecer demasiado académica ou hermética. No entanto, não efectuar este exercício é, nos dias de hoje, ainda mais redutor. De facto, a forma como as universidades (e a respectiva investigação) se vão orientar no futuro está claramente relacionada com uma divisão entre as que serão consideradas de investigação e aquelas cuja vertente principal será o ensino.

O presente estudo pretende efectuar uma análise crítica do ranking das instituições ibero-americanas proposto pela SCImago [3], com base na produção científica dessas instituições. Trata-se de um exemplo de abordagem que se pretende objectiva e que tenta evitar uma complexa e possivelmente inconclusiva incursão no mundo dos também designados "rankings" (QS, Times, etc.). Têm surgido vários, baseados em características, algoritmos e propósitos bem distintos e com óbvias dificuldades de validação. Refira-se aqui que a subtil variação nos significados da palavra "ranking", que vai desde simples ordenação, posicionamento, até lista ordenada ou metodologia para a sua construção contribui para alguma falta de clareza na exposição. No que se segue, far-se-á um esforço para que não haja ambiguidade.

Note-se que existem consequências práticas do posicionamento nas listas de impacto institucional. A ordenação 
baseada num critério único de produtividade em bruto relativo ao quinquénio 2007-2011 parece ter contribuído para a decisão de suspensão do programa "Ciência sem Fronteiras” (CsF) para Portugal [4]. Mostrar-se-á neste estudo que a ordenação efectuada por uma qualquer metodologia deve ser cuidadosamente analisada e deve, optimamente, incluir critérios de qualidade e produtividade.

\section{QuANTIDADE E QuALIDADE}

O conjunto de universidades analisadas é constituído pelas 50 primeiras instituições do ranking ibero-americano SCImago relativo ao período 2007-2011. O Instituto Superior Técnico, não contemplado no estudo SCImago, é também considerado, dada a sua relevância a nível das 50 universidades analisadas. O universo de universidades/ institutos passa assim a ser de 51 .

Os dados sobre os quais a SCImago actua é proveniente da base de dados Scopus, enquanto que os dados recolhidos para análise advêm da base de dados ISI Web of Knowledge (WoK). No WoK discriminam-se duas vias para obter o conjunto de dados: (i) pesquisa do número de publicações científicas no ISI Web of Science, no mesmo período da SCImago (2007-2011), (ii) identificação das áreas de ranking ESI (Essential Science Indicators) de cada instituição, número de artigos e número de citações para cada área de ranking, bem como o número total de artigos e citações da instituição no campo “All Fields” (consulta ESI a 1 de Maio de 2013). O facto da WoK permitir recolher, de um modo fácil, esta informação promoveu o seu uso no presente estudo.

A Tabela 1 apresenta os resultados obtidos para a Universidade de Coimbra (UC). De acordo com a metodologia proposta, a UC possui 11 áreas de ranking. No período de 1 de Janeiro de 2003 a 28 de Janeiro de 2013, “Chemistry” é a disciplina que apresenta o maior número de artigos (ca. de 1600) e também maior número de citações (14809). Durante este período, a UC publicou 8945 artigos (“All

Tabela 1 - Número de artigos e citações correspondentes às áreas de ranking da Universidade de Coimbra no período de 1 de Janeiro de 2003 a 28 de Janeiro de 2013 (consulta ESI a 1 de Maio de 2013)

\begin{tabular}{|l|r|r|}
\hline \multicolumn{1}{|c|}{ Áreas Rk } & Artigos & Citações \\
\hline 1. Chemistry & 1600 & 14809 \\
\hline 2. Physics & 1005 & 9872 \\
\hline 3. Clinical Medicine & 948 & 9165 \\
\hline 4. Neuroscience \& Behavior & 359 & 7682 \\
\hline 5. Biology \& Biochemistry & 456 & 6143 \\
\hline 6. Engineering & 1028 & 5304 \\
\hline 7. Pharmacology \& Toxicology & 312 & 4451 \\
\hline 8. Environment \& Ecology & 434 & 3811 \\
\hline 9. Plant \& Animal Science & 491 & 3669 \\
\hline 10. Materials Science & 519 & 3151 \\
\hline 11. Agricultural Science & 111 & 1066 \\
\hline All Fields & 8945 & 80877 \\
\hline
\end{tabular}

Fields”), com um total de 80877 citações. Tal corresponde a perto de 10 citações por artigo, um número característico de grande prevalência.

Com base na metodologia usada (dados ESI e WoS) é possível fazer uma análise crítica dos dados da SCImago. De facto, numa primeira fase podemos comparar os dados brutos, relativos ao número de publicações, introduzindo também o número de áreas no ranking, que designaremos por $R k^{1}$ [5]. A Tabela 2 apresenta como exemplo o número de publicações SCImago vs. número de publicações WoS, para as 30 primeiras universidades do ranking SCImago. Nesta tabela é igualmente contabilizado o $R k$ para cada uma das instituições. Por comparação, verifica-se que as cinco primeiras universidades do ranking SCImago permanecem na mesma posição utilizando o que podemos designar por “ranking WoS”. Este resultado, valida a comparação entre o ranking SCImago e a WoK, em termos de número de artigos e mostra também que a quantidade é necessariamente geradora de qualidade, não sendo no entanto o critério único ou mesmo determinante. No entanto, da observação da Tabela 2 resultam outros dados merecedores de relevo. As universidades de grande dimensão, consideradas como “Universidades Clássicas”, abrangem um grande número de áreas científicas, apresentando $R k$ ’s iguais ou superiores a 15, o que acompanha igualmente o ranking de produtividade em bruto. De facto, as 10 primeiras universidades surgem com $R k=15$. A excepção é a Universidade Estadual Paulista Júlio de Mesquita Filho com $R k=12$. Por outro lado, universidades com $R k=17$, como as Universidades de Valência e Autónoma de Madrid surgem no ranking SCImago em posições abaixo das 10 primeiras. Este facto sugere que os critérios de qualidade são de algum modo subvalorizados quando é efetuada uma análise exclusivamente baseada no número de artigos, como é o caso da ordenação resultante do ranking SCImago. No entanto, é importante salientar que a análise feita pela SCImago contém parâmetros diferenciadores indicadores de qualidade. Um desses parâmetros de qualidade é definido pela fracção do número de publicações que se encontram no primeiro quartil das revistas com maior impacto e designado por Q1 e que assenta na fracção de artigos publicados em revistas pertencendo ao primeiro quartil numa determinada categoria científica, e ordenadas pelo indicador SJR (SCImago Journal Rank). Porém, a ordenação das instituições resultante no ranking é normalmente estabelecida com base apenas no número de artigos.

\section{Quantidade e Dimensão}

O índice SCImago é geralmente utilizado para dispor as instituições de investigação por ordem de número de publicações. Este parâmetro tem a vantagem de permitir a comparação directa, e podemos aceitar que uma instituição que publica maior número de artigos está associada a um maior impacto dentro da comunidade científica. Outros parâmetros constantes deste índice incidem sobre a quali-

\footnotetext{
1 O ESI organiza as áreas científicas de cada instituição (Field Rankings) que se localizam em citações no $1 \%$ de topo a nível mundial na respectiva área, aquilo que será aqui denominado por $R k$
} 
Tabela 2 - Número de publicações SCImago vs. WoS, para as 30 primeiras universidades do ranking SCImago. O número de áreas de ranking ESI é também contabilizado. Os números indicados à esquerda da instituição reportam-se à ordenação do ranking SCImago para o período de 2007-2011

\begin{tabular}{|c|c|c|c|c|}
\hline Instituição & $\begin{array}{l}\text { \#Artigos } \\
\text { SCImago }\end{array}$ & $\begin{array}{c}\text { \#Artigos } \\
\text { WoS }\end{array}$ & $\begin{array}{c}\text { Alteração } \\
\text { posição }\end{array}$ & \#Áreas $\boldsymbol{R} k$ \\
\hline 01-Univ São Paulo & 47833 & 44777 & $=$ & 19 \\
\hline 02-Univ Nacional Autónoma México & 19349 & 17648 & $=$ & 18 \\
\hline 03-Univ Barcelona & 16914 & 16815 & $=$ & 19 \\
\hline 04-Univ Estadual Campinas & 16885 & 14996 & $=$ & 15 \\
\hline 05-Univ Estadual Paulista Júlio de Mesquita Filho & 16810 & 14277 & $=$ & 12 \\
\hline 06-Univ Fed Rio de Janeiro & 14702 & 12860 & -2 & 17 \\
\hline 07-Univ Autónoma Barcelona & 14576 & 13506 & +1 & 19 \\
\hline 08-Univ Complutense Madrid & 14351 & 13101 & +1 & 18 \\
\hline 09-Univ Porto & 12904 & 10790 & -2 & 16 \\
\hline 10-Univ Fed Rio Grande do Sul & 12223 & 11290 & +1 & 15 \\
\hline 11-Univ Politécn Catalunha & 12010 & 8188 & -6 & 7 \\
\hline 12-Univ Valência & 11928 & 11281 & +2 & 17 \\
\hline 13-Univ Autónoma Madrid & 11678 & 10129 & +1 & 17 \\
\hline 14-Univ Técn Lisboa & 11553 & 8186 & -4 & 6 \\
\hline 15-Univ Buenos Aires & 11452 & 9808 & +2 & 16 \\
\hline 16-Univ Fed Minas Gerais & 10711 & 9478 & +1 & 13 \\
\hline 17-Univ Granada & 10285 & 9589 & +3 & 14 \\
\hline 18-Univ Politécn Valencia & 9441 & 7453 & -3 & 7 \\
\hline 19-Univ Fed São Paulo & 9433 & 9199 & +3 & 7 \\
\hline 20-Univ Sevilha & 8861 & 7316 & -3 & 13 \\
\hline 21-Univ Politécn Madrid & 8760 & 5515 & -3 & 7 \\
\hline 22-Univ Zaragoça & 8504 & 7493 & +2 & 11 \\
\hline 23-Univ Chile & 8354 & 7889 & +4 & 12 \\
\hline 24-Univ País Vasco & 8312 & 7433 & +2 & 7 \\
\hline 25-Univ Santiago de Compostela & 7507 & 6388 & $=$ & 13 \\
\hline 26-Univ Lisboa & 7220 & 5613 & -4 & 10 \\
\hline 27-Univ Coimbra & 7162 & 6902 & +3 & 11 \\
\hline 28-CINVESTAV IPN & 7072 & 5792 & +1 & 3 \\
\hline 29-Univ Aveiro & 6743 & 5837 & +3 & 8 \\
\hline 30-Univ Fed Santa Catarina & 6521 & 5333 & -3 & 9 \\
\hline Inst SuperTécn ${ }^{\mathrm{a}}$ & & 5775 & & 5 \\
\hline
\end{tabular}

a O Instituto Superior Técnico, não contemplado no estudo SCImago, é também considerado, dada a sua relevância a nível das 50 universidades analisadas.

dade da produção, por exemplo um já antes referido (Q1) e que assenta na fracção de artigos publicados em revistas pertencendo ao primeiro quartil. Este tipo de parâmetros parece sugerir uma combinação com o correspondente à produção global, permitindo-nos combinar qualidade e quantidade. Naturalmente, que uma forma directa de proceder corresponde a multiplicar a fracção da produção de qualidade pela produção total, originando o total de produção de qualidade. Trata-se de um indicador que, naturalmente, dá conta também da dimensão da instituição e é fácil de perceber que se todas as instituições tivessem, por hipótese, o mesmo Q1, as maiores apareceriam nos lugares cimeiros do ranking. A discriminação que resta é, portanto, a das grandes instituições com baixo Q1 e a das pequenas com elevado Q1, mas o impacto na ordenação é normalmente pequeno. A ordenação directa através deste indicador é ainda mais problemática, porque existem instituições diminutas, com produtividade quase nula mas de alto impacto que surgem, destacadas, no topo da tabela.

Vemos, assim, a necessidade de normalizar a produção (global ou de qualidade) com grandezas de outro tipo, como já defendido anteriormente em diferentes trabalhos $[6,7]$. A mais óbvia será naturalmente o número de docentes ou de investigadores, mas o financiamento poderá ser uma alternativa. Note-se, no entanto, que esta última peca pelo facto de ser difícil de estabelecer em estudos efectuados a partir do exterior da instituição. O mesmo sucede com o número de investigadores, que é difícil de avaliar. O número de docentes numa universidade portuguesa pode ser determinável, mas possivelmente já se torna mais complexo determinar o número de pós-doutorados englobados 
nos diversos programas ou projectos. Existe a esperança de resolver tal dificuldade com base em plataformas tipo DeGóis (Portugal) ou Currículos Lattes (Brasil), mas estender tal aproximação para instituições arbitrárias em qualquer zona do planeta parece mais difícil. No entanto, estratégias para recolher o máximo de informação têm de estar na ordem do dia, especialmente num país em que os nossos departamentos de investigação são, tipicamente, de dimensões reduzidas e, portanto, a sua qualidade só pode ser aferida se normalizada pelo número de pessoas envolvidas. E, acrescentamos, pelo financiamento atribuído às mesmas instituições.

\section{Considerações FinAis}

A maior parte dos procedimentos de ranking estão associados à produção global, com ou sem avaliação da respectiva qualidade, e portanto dependem da dimensão institucional. Portugal ficará, assim, tipicamente mal colocado face a países com maiores populações e universidades com maior número de docentes e alunos (vejam-se as Universidades de S. Paulo ou de Barcelona, acima, de dimensões cerca de 5 e 2 vezes, respectivamente, maiores que a da Universidade de Coimbra, por exemplo). Ficará ainda mais mal colocado face a instituições de investigação de nível (global) nacional, como sejam o CNRS, o CSIC ou o Max Planck Institute, algumas delas a atravessar presentemente forte crise.

Torna-se, pois, necessário crescer, mas torna-se também necessário normalizar. Produzimos com investigadores que devem ser contabilizados, como contabilizados devem ser os dos pares estrangeiros com que nos queremos comparar. Temos também de caracterizar as nossas instituições, e parâmetros como o $R k$ podem ser extremamente úteis para, por exemplo, julgar da abrangência. Só a inspecção deste parâmetro levaria imediatamente à conclusão que deveria ser tentada a junção da Universidade Técnica com a Clássica, em Lisboa. O que foi feito!

\section{REFERÊNCIAS}

[1] J. E. Hirsch, “An index to quantify an individual's scientific research output”, PNAS 102 (2005)16569-16572

[2] Notícia Público, 25 de Julho de 2005 (fonte: http://www. publico.pt/educacao/noticia/aveiro-lidera-investigacaouniversitaria-em-portugal-1229006)

[3] http://www.scimagoir.com e http://scimagoir.com/country/

[4] Notícia Estadão.com.br, 5 de Março de 2013 (fonte: http:// www.estadao.com.br/noticias/impresso,bolsistas-fazemcurso-pior-emportugal,1004388,0.htm)

[5] T.F.G.G Cova, A.A.C.C. Pais, S.J. Formosinho, "Iberian universities: a characterisation from ESI rankings”, Scientometrics 94 (2013)1239-1251

[6] E.S. Vieira, J.A.N.F. Gomes, “The journal relative impact: an indicator for journal assessment”, Scientometrics $\mathbf{8 9}$ (2011) 631-651

[7] E.S. Vieira, J.A.N.F. Gomes, “An impact indicator for researchers”, Scientometrics 89 (2011) 607-629

\section{Antiguidades Científicas}

\section{Moléculas Natalícias}

O ácido boémico é uma mistura de compostos químicos com propriedades anti-tumorais cujos nomes derivam de personagens da ópera La Bohème (1896) de Giacomo Puccini. Assim, esta mistura inclui compostos como marcelomicina (Marcello), musettamicina (Musetta), rudolfomicina (Rodolfo), mimimicina (Mimí), collinemicina (Colline), alcindoromicina (Alcindoro) e schaunardimicina (Schaunard).

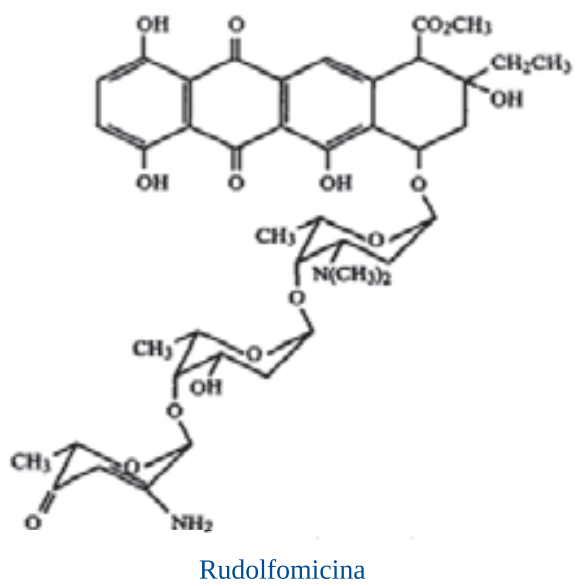

Atente-se na subtileza de rudolfomicina, que não deriva exactamente do nome do personagem Rodolfo de $L a$ Bohème mas antes de Rudolph, the Red-Nosed Reindeer. Com efeito, por degradação da rudolfomicina obtém-se um açúcar, correspondente ao da extremidade da molécula de origem, o qual foi designado por rednose.

Este nome, algo insólito para um composto químico, talvez possa ser explicado se atendermos a que o manuscrito

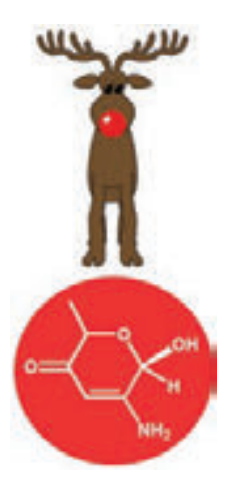
original foi submetido ao Journal of the American Chemical Society no dia 21 de Dezembro de 1978... em plena época natalícia.

(adaptado de http://www.chm.bris.ac.uk/ sillymolecules/sillymols2.htm e de J. Am. Chem. Soc. 101 (1979) 7041-749)

João Paulo André (jandre@quimica.uminho.pt) 